\title{
Rate and frequency as determinants of learning with complete and discrete list presentation
}

\author{
JAMES W. HALL, TODD A. SMITH, SUZANNE L. WEGENER, \\ and BENTON J. UNDERWOOD \\ Northwestern University, Evanston, Illinois 60201
}

\begin{abstract}
In Experiment 1, complete presentation of the study list produced better free recall learning than did the usual item-by-item (discrete) presentation. The difference was large and held for items occurring one, two, or three times within a list, whether items were spaced or massed, and for discrete presentation rates of 2, 4, and 6 sec/item. Experiment 2 replicated this superiority of complete over discrete presentation (equating total study time), and Experiment 3 extended the finding to paired associate learning. Experiments 2 and 3 indicated that multiple presentations of a list at fast rates were superior to a single presentation at a more standard rate and only slightly inferior to a single, complete presentation. Practical implications for instruction were pointed out, as were problems that certain of the results pose for theories that emphasize strategic (or at least extended) processing of items for encoding.
\end{abstract}

At some point in the distant past, investigators of learning and memory began to use discrete presentation of the materials to be learned. The items forming a list of words or a list of nonsense syllables were presented one at a time for study, perhaps by having each item on an index card, or perhaps by the use of a memory drum or a slide projector. Each item was presented successively for a few seconds, with $2 \mathrm{sec}$ probably being the modal time for each item across studies. Most of us never gave the matter a thought; to present materials for learning in an experiment meant discrete presentation.

Discrete presentation presumably has some merits, in that the subjects are given the same amount of time to study each item, although few investigators would deny that subjects may rehearse items other than the one being shown at the moment. Whatever, our laws of learning and memory are laws of discrete presentation. Discrete presentation is in contrast to complete presentation, wherein the subjects are given the entire list on a sheet of paper and are allowed a given amount of time to study the items.

The question of interest is why anyone would choose to use complete presentation. A variety of answers could be given, some of which will be mentioned here. It could be argued that complete presentation has higher ecological validity than does discrete presentation. Being ecologically more valid, complete presentation allows the subjects greater freedom in using their skills to study the items in a manner they choose. Perhaps a more critical answer to the question revolves around the phenomenon of organization. It is common today to place heavy emphasis on organization as a factor in learning and memory. It would seem that most theorists would accept the idea that subjects could more readily organize their learning when presentation is complete than when it is discrete. If organization is a pivotal notion, it must follow that complete presentation would be superior to discrete presentation in free recall learning. By like reasoning, it would seem to follow that if the task were one for which organization would not aid learning (or would aid it minimally), complete presentation would not be superior to discrete presentation, although we realize that the discrete-complete variable might influence learning for reasons not related to organization. Nevertheless, paired associate and serial learning, for example, should not be influenced much by opportunity for organization, and the effect of the discrete-complete variable should be less than it is with a free recall task. What we wish to emphasize at this time is that the variable of interest has a substantial potential for testing theoretical notions. The role of organization in learning and memory seems to be one such notion.

Our search of the literature has shown that experiments in the past have produced a very mixed set of results when the discrete-complete variable has been manipulated. Some studies have shown that performance is better with complete than with discrete presentation. In this group would be studies by Foote and Pollio (1970), who studied free recall using common words, Hall, Grossman, and Elwood (1976), who also used common words in free recall and recognition, and Moursund and Chape (1966), who used paired associates consisting of nonsense syllables. Unfortunately, this latter finding was directly contradicted by the results reported by Brown and Read (1966). Brown and Read found that the complete method was markedly inferior to the discrete method when paired associate learning of nonsense syllables was involved. Several studies have shown no differences resulting from comparisons of the discrete and complete (Knox \& Wolf, 1965; Lauer, Streby, \& Battig, 1976; Light \& Schurr, 1973; Mueller, Rankin, \& Carlomusto, 1979).

There are intermediate steps between discrete and 
complete presentation. Thus, if there are 12 words in a list, they might be presented as six sets of 2 items, three sets of 4 , or two sets of 6 , with, of course, the total study time for all 12 items being held constant. Imhoff, Horton, Weldon, and Phillips (1977) used such manipulations (but not complete presentation) with both words and nonsense syllables and found that as set size increased, performance decreased. Brown and Brown (1965) report the same finding.

It might be surmised that if organization is involved in the effects of the discrete-complete variable, maximum differences in favor of complete presentation would be found when related words are used in a free recall list. Two studies have found no effect of this manipulation (Foote \& Pollio, 1970; Mueller et al., 1979), and one showed that discrete presentation gave faster learning than did presentation of groups of items that were not complete (Imhoff et al., 1977). Such data are difficult to rationalize if organization is a critical factor in learning. Furthermore, the fact that the complete method has not been found to be consistently better than the discrete method does not seem to be consonant with an organizational emphasis of learning.

The complete vs. discrete issue is of central concern in the three experiments reported here. A second central issue is the relative efficacy of variations in the rate and frequency of presentation of items using the discrete format. The issues are related in that the relative effectiveness of complete and discrete presentation may depend on such factors as the rate of discrete presentation and whether items or lists are repeated or presented a single time. In fact, such variables might account for some of the contradictory findings regarding the complete-discrete variable that we have reviewed above.

The question of relative effectiveness of various rates and frequencies of presentation also is a significant one in its own right. There are, for example, potential educational implications of identifying optimal presentation rates for particular classes of materials and particular task demands. Moreover, there may be important theoretical implications as well. As noted earlier, current theoretical notions regarding organization seem to imply the superiority of certain study conditions. In particular, one would expect that list organization by the learner would be difficult with rapid presentation rates. Formulations that emphasize extended "elaboration" of individual items (e.g., Anderson \& Reder, 1979) would seem to lead to similar expectations. In fact, any theoretical formulation in which heavy emphasis is placed on the importance of extensive strategic processing of items during encoding seems to imply that considerable time will be required to complete that processing. The amount of time for such encoding has not been examined in detail empirically, nor has any precise estimate been offered. However, it is difficult to imagine that very much strategic elaboration or organization would be possible when items are presented at as rapid a rate, say, as $1 \mathrm{item} / \mathrm{sec}$. Thus, if recall under rapid rates of presentation were as good as or better than with the usual slower rates, one would be forced to reexamine those formulations in which elaborative encoding or organization is held to be critical for high levels of item recall.

There are some data available that bear on this presentation rate question, but, as with the completediscrete literature, they do not permit any clear-cut conclusion at this point. One set of studies suggests that, within a fairly wide range of values, presentation rate is of little consequence, provided total study time is held constant (e.g., Brewer, 1967; Bugelski, 1962). In contrast, Johnson (1964) reported differences in learning rate as a function of presentation rate (and, therefore, number of exposures) with total study time held constant, and Nodine (1965) and Stubin, Heimer, and Tatz (1970) reported superior learning with very rapid presentation time. However, all of the above studies examined paired associate learning, and the materials used were nonsense items. This, together with the conflicting findings of the studies, provides little basis for firm conclusions, particularly with respect to free recall.

There are two empirical generalizations that bear on the differential rate question. One is the total time hypothesis (Cooper \& Pantle, 1967), which would lead to the expectation of no substantial differences as a function of presentation rate, provided that the total amount of study per item is held constant. If that generalization were to hold for the case of a single-list repetition, using rates as rapid as $1 \mathrm{sec} / \mathrm{item}$, it would seem to put some strain on any theoretical position in which strategic elaborative or organization processing is central. The second empirical principle that is pertinent here is the well established spacing effect (Melton, 1970), in which the distribution of repetitions within a list produces better recall than when those repetitions are massed. Extending that principle to the present situation, one might expect better performance with the faster rates because what one has when the list is repeated, in effect, is a single list in which items are widely distributed. The single, slower rate presentation may be conceptualized as a massed condition, in that the exposure of an item for $4 \mathrm{sec}$, say, is roughly equivalent to four consecutive 1 -sec exposures. (This latter equivalence has not been established empirically.) Thus, analyzing the situation as one in which number of exposures is varied leads to the expectation of higher performance with the $1-\mathrm{sec}$ than with the 4-sec rate, a result that would seem even more problematic for an elaboration or organization formulation of effective memory encoding.

\section{EXPERIMENT 1}

In our first experiment, both the mode of presentation (complete vs. discrete) and study times were varied. The times per item for discrete presentation were 
2,4 , and $6 \mathrm{sec}$. The words were presented by a slide projector, and total time was calculated as exposure time plus slide-change time. For complete presentation, the times corresponding to 2,4 , and $6 \mathrm{sec}$ for discrete presentation were 88,150 , and $212 \mathrm{sec}$. We assumed that in discrete presentation, the subjects make complete use of slide-change time $(.85 \mathrm{sec})$ for study and rehearsal. If this assumption is not true, effective study time will be greater for complete than for discrete presentation. It may be mentioned at this time that in the previous studies on the discrete-complete variable, it has not always been clear what constitutes the total time. It may be that some of the discrepancies in the findings are due to the fact that the change time or off time between items has not always been considered a part of total time.

As a third variable, we have included spacing of repeated items as a within-subjects variable. We accept the fact that with discrete presentation, spaced items will give higher recall than will massed items. What is not clear is what will be the outcome when complete presentation is used. In particular, it is difficult to analyze the situation for a massed item. One question is whether the subjects will give as much study time to a massed item in complete presentation as they would to a massed item in discrete presentation. If not, one would expect the spacing effect to be greater for complete presentation than for discrete. However, if under complete presentation the subjects "recirculate" through the list, the massed items also would receive distributed rehearsal, with a consequent reduction in the spacing effect. There seemed to be no clear basis for a prediction.

\section{Method}

Conditions. We have identified the six basic conditions above. With discrete presentation, the rate of presentation was 2,4 , and $6 \mathrm{sec}$, and with complete presentation, the corresponding times were 88,150 , and 212 sec.

Lists. Each subject was given a single study and test trial on each of eight successive lists. The results for the eight lists were summed to produce the desired stability. The words were all five-letter nouns and varied in frequency from 10 per million to A frequencies (Thorndike \& Lorge, 1944). They were assigned to lists and to a function within lists on a random basis. Each list contained 19 words. Of these, two were primacy buffers and three were recency buffers. Six words occurred once each in the body of the list, and all remaining positions were occupied by massed and spaced items. Two words occurred twice each as massed items and two occurred twice each as spaced items. A total of 31 positions was required for each list. Four different list structures were used to prevent the subjects' anticipating particular patterns of repetition, with two lists using each structure. The eight lists were presented in the same order to all subjects.

Procedure and Subjects. A slide projector was used to present the list under discrete presentation. For complete presentation, the 31 words were typed in a single column, double spaced, in the middle of a sheet of paper. The sheets were presented face down to the subjects, who then turned the sheet over at a signal from the experimenter to start the study of the list. The sheets were removed when the study time had expired. The subjects were fully instructed about the number of lists, and recall was recorded in an eight-page booklet. Recall time for each list was limited to $90 \mathrm{sec}$.
The six conditions were represented by six groups of 36 subjects each. The college student subjects were tested in groups of six, and the condition assigned to a particular group of six subjects was determined by a prearranged block-randomized schedule. Because some subjects failed to appear at the scheduled time, some of the groups did not initially contain six subjects. It was necessary to go through the schedule a second time to pick up the missing subjects.

\section{Results and Discussion}

We will first examine the total recall as a function of study time and the discrete-complete variable. The results, in terms of mean correct per list, are seen in Figure 1. Two findings are obvious. First, recall was better for complete than for discrete presentation. Second, recall increased as study time increased between 2 and $4 \mathrm{sec}$ and between 88 and $150 \mathrm{sec}$, but additional time had no effect for either method. Using the $5 \%$ significance level, we found that both findings would be judged statistically reliable [for method, $F(1,210)=$ 24.99, $\mathrm{MSe}=487.45$, and for study time, $\mathrm{F}(2,210)=$ 19.80 , with the same mean square). The superiority of complete over discrete held for items occurring once within the body of the list and for the spaced and massed items, but not for the primacy and recency buffers, which were recalled equally often (statistically speaking) under the two methods.

A comparison of the recall of massed and spaced items is given in Figure 2. Frequency is plotted along the baseline, with percent recall on the ordinate. The data have been summed across study time because there was no interaction between study time and massed vs. spaced practice. A large spacing effect was found, as expected, for the discrete presentation, with a smaller but still distinct effect for the complete presentation.

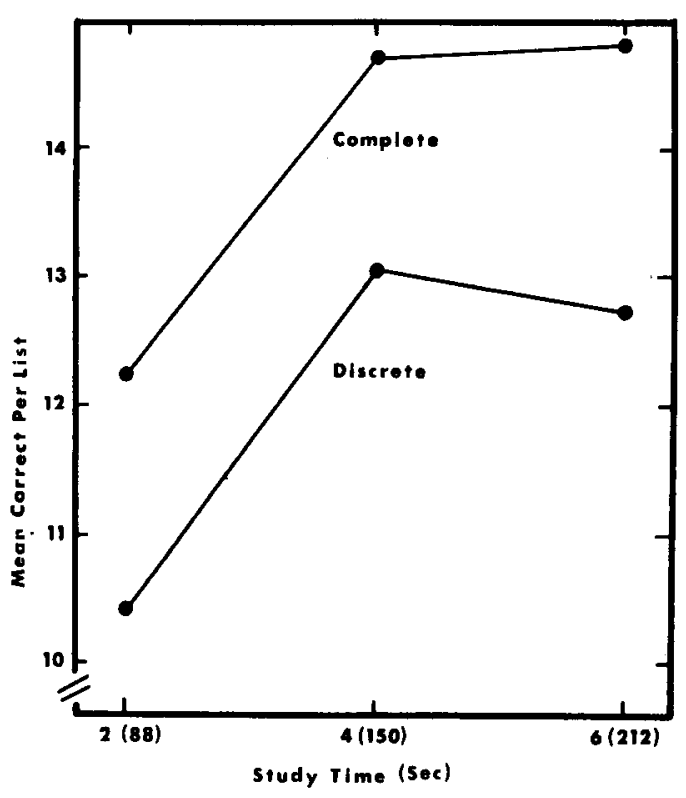

Figure 1. Mean recall per list as a function of study time and of discrete and complete presentation. 


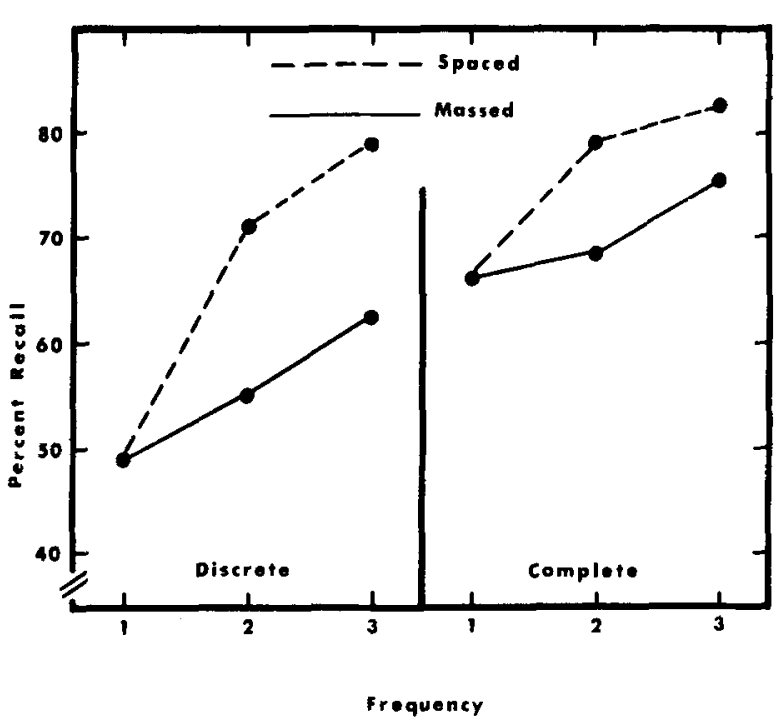

Figure 2. Percent recall as a function of spaced vs. massed practice and discrete vs. complete presentation.

There was statistical reliability for the difference in the magnitude of the spacing effect for the two methods $[F(1,210)=25.00, \mathrm{MSe}=12.23]$ when the spacing effect was determined for each subject by subtracting the recall of all massed items from the recall of all spaced items.

Several analyses were done involving stage of practice, using performance on the first four lists vs. performance on the last four lists. Performance did increase a small amount across lists, but we did not find any interactions with the other variables.

Our results for the discrete-complete variable were unambiguous; at all study intervals, the superiority of the complete presentation over the discrete was obvious. However, the interpretation of these differences may seem to be somewhat ambiguous. It will be remembered that for discrete presentation, the slide-change time was included as a part of the total time when calculating the times to be used for complete presentation. It might be argued that in discrete presentation, the subjects do not in fact use the change time for study and that the differences observed in Figure 1 between the two methods are due to differences in total study time. Four arguments may be advanced against this interpretation.

(1) It does not seem possible that the blank period between slides is also always a blank period of the same length in the subjects' rehearsal and encoding activities. Surely, many subjects use the slide-change time to rehearse.

(2) Let it be assumed that the subjects did not use slide-change time to rehearse at all. We could then calculate how many correct responses should have been given had slide-change time been used for study (assuming that the amount learned in slide-change time was at the rate per unit of time as that observed). These cal. culations indicated that amount learned under the discrete procedures would have still fallen short of that for complete presentation.

(3) If the differences between complete and discrete methods were due to subjects' not using slidechange time for study in the discrete method, the differences in learning between complete and discrete should have decreased as exposure time increased from 2 to 4 to $6 \mathrm{sec}$. Change time remained constant at $.85 \mathrm{sec}$ as total exposure time increased. Hence, the proportion that change time was of total time decreased as total exposure time increased $(.43, .21$, and .14 , for exposure times of 2, 4, and $6 \mathrm{sec}$, respectively). As is obvious in Figure 1, the differences between recall under the discrete and complete methods did not change in the way expected if a difference in study time resulted from the failure of the subjects to use slide-change time for study.

(4) Experiments 2 and 3, to which we will turn shortly, control change time between items, and the complete method remained superior to the discrete method.

We must express puzzlement at our data, in which performance did not improve as study time increased from 4 to $6 \mathrm{sec}$ with discrete presentation and from 150 to $212 \mathrm{sec}$ with complete presentation. We could cite many, many sets of data in which recall and study time are directly related, but we cannot cite a single study that shows results like those pictured in Figure 1 for discrete presentation. We have not been able to find a reasonable explanation for the lack of effect of study time beyond $4 \mathrm{sec}$.

\section{EXPERIMENTS 2 AND 3}

As in Experiment 1, Experiment 2 examined free recall under complete and discrete presentation conditions. The principal difference between the experiments was that Experiment 2 examined two rates of discrete presentation, holding total presentation (study) time constant. That is, for one discrete presentation condition, the list was presented a single time at a relatively slow (4-sec) rate, whereas for a second condition, the list was presented four times at a fast $(1-\mathrm{sec})$ rate.

Experiment 3 was conducted to examine the generality of presentation method effects to the paired associate task. Because the two experiments employed the same subjects and basic design and were run within the same session, they are reported together here.

In each experiment, all subjects served in each of the three presentation conditions: complete, discrete fast, and discrete slow. Two lists were presented successively under each condition, and the order of conditions was completely counterbalanced. In the paired associate study (Experiment 3), half the subjects were tested by using a complete procedure and half using a discrete procedure. All items were presented visually by com- 
puter, eliminating the rather knotty problem of change time that occurs when slide projectors or memory drums are used.

\section{Method}

Subjects. The subjects in both experiments were 24 undergraduate students, 23 of whom received credit toward completion of their introductory course in psychology. One was an outright volunteer.

Design and Procedure. In each experiment, the subjects received six consecutive study lists presented, along with instructions, on the video screen of an Apple II microcomputer. There were three conditions of study presentation, with two lists per condition. In the complete condition, the items were displayed simultaneously in two columns on the screen for $96 \mathrm{sec}$. In the discrete fast condition, the items were presented successively on the center of the screen at a 1-sec rate for the 24-item lists in Experiment 2 and at a 2 -sec rate for the 16-item lists in Experiment 3. The total list was presented four times in immediate succession in the discrete fast condition in Experiment 2 and three times in the discrete fast condition in Experiment 3, for a total of $96 \mathrm{sec}$ of study time per list in both cases. (All rates refer to exposure time; change time or off time was negligible.) In the discrete slow condition, each list was presented a single time and items were presented successively in the center of the screen at a 4-sec rate for free recall (Experiment 2) and at a 6-sec rate for paired associate learning (Experiment 3 ), again for a total of $96 \mathrm{sec}$ for each list. The subjects were randomly assigned to the six conditions necessary for a complete counterbalancing of presentation condition order. The order of particular lists remained constant across subjects.

Immediately following the presentation of each list for study in Experiment 2, a free recall period of $108 \mathrm{sec}$ was given, in which the subjects recorded items in a test booklet. A 12-sec delay occurred between the end of recall and the presentation of the next list. The paired associate testing procedure was similar, except that for half the subjects (randomly assigned), the stimulus items appeared successively in the center of the screen at a 6-sec rate (the discrete test) and for the remaining subjects, the total list of stimulus items appeared for $96 \mathrm{sec}$ (the complete test). Again, responses were recorded by the subject in a test booklet.

For every subject, the two experiments were conducted within the same $1-\mathrm{h}$ session, with the free recall experiment first and a break between experiments just long enough to present the new instructions. Before the first list was presented in each experiment, the three presentation rates were illustrated, using a brief sample list.

Materials. The free recall lists were formed by randomly assigning 144 of the 152 words used in Experiment 1 to the six lists of 24 words each for Experiment 2. The pairs used in Experiment 3 consisted of rare English nouns (e.g., ARMAGER) as stimuli and familiar nouns (e.g., PLOW) as responses. From a pool of 96 pairs, words were assigned randomly to the six lists.

\section{Results and Discussion}

Experiment 2. The free recall data are summarized in the top portion of Table 1 . Note that recall was highest for the complete condition and lowest for the discrete slow condition and that recall was considerably better for the second of the two lists within each condition. Both of these main effects were significant in a presentation method by list (first vs. second) by order ANOVA $[\mathrm{F}(2,36)=11.11, \mathrm{MSe}=6.76$, and $\mathrm{F}(1,36)=$ $26.53, \mathrm{MSe}=4.63$, respectively]. The only other significant effect was the triple interaction $[F(10,36)=2.44$, $\mathrm{MSe}=4.44]$, which suggested that particular sequences of presentation conditions resulted in greater differences between the first and second lists than did others, and which probably does not warrant interpretation, given the very small $\mathrm{ns}$ in the cells involved.

These results rather clearly replicate those of Experiment 1 in demonstrating the superiority of complete to 4-sec discrete presentation. This replication was important, because the use of the computer for presentation in Experiment 2 eliminated the problem of how time between exposures of the words in discrete presentation (change time) should be allocated (i.e., whether or not it should be considered as study time). For practical purposes, there was no change time in Experiment 2 and 3. Thus, this factor was eliminated as a source of performance differences among the study conditions in these experiments.

Although the overall ANOVA yielded the anticipated significant effect of presentation method, it did not fully address the two principal questions of concern. These were (1) whether the two discrete conditions differed in their effects on recall and (2) whether the complete condition was superior to the discrete fast condition, in which there were multiple presentations of the list. To answer these questions, simple comparisons between the relevant conditions were made using $t$ tests. These comparisons were made combining the first and second lists within the study condition and again for the second lists only. The decision to make the latter group of comparisons was based on the fact that performance improved markedly from the first to the second lists, especially within the discrete fast conditions, suggesting that second list performance may provide a more stable estimate of performance.

Table 1

Mean Recall (and Standard Deviations) for the Two Experiments as a Function of Complete Presentation and Slow and Fast Discrete Presentation

\begin{tabular}{|c|c|c|c|c|c|c|c|c|c|c|c|c|}
\hline \multirow{3}{*}{$\begin{array}{l}\text { Exper- } \\
\text { iment }\end{array}$} & \multicolumn{4}{|c|}{ Complete } & \multicolumn{4}{|c|}{ Discrete Fast } & \multicolumn{4}{|c|}{ Discrete Slow } \\
\hline & \multicolumn{2}{|c|}{ List 1} & \multicolumn{2}{|c|}{ List 2} & \multicolumn{2}{|c|}{ List 1} & \multicolumn{2}{|c|}{ List 2} & \multicolumn{2}{|c|}{ List 1} & \multicolumn{2}{|c|}{ List 2} \\
\hline & Mean & SD & Mean & $\overline{\mathrm{SD}}$ & Mean & SD & Mean & SD & Mean & SD & Mean & SD \\
\hline $\begin{array}{l}1 \\
2\end{array}$ & $\begin{array}{r}14.67 \\
8.75\end{array}$ & $\begin{array}{l}6.14 \\
4.11\end{array}$ & $\begin{array}{l}15.83 \\
10.38\end{array}$ & $\begin{array}{l}4.74 \\
3.55\end{array}$ & $\begin{array}{r}12.13 \\
8.79\end{array}$ & $\begin{array}{l}4.06 \\
3.74\end{array}$ & $\begin{array}{r}14.96 \\
9.88\end{array}$ & $\begin{array}{l}4.30 \\
4.19\end{array}$ & $\begin{array}{r}12.04 \\
7.04\end{array}$ & $\begin{array}{l}3.35 \\
4.06\end{array}$ & $\begin{array}{r}13.58 \\
8.71\end{array}$ & $\begin{array}{l}4.33 \\
3.75\end{array}$ \\
\hline
\end{tabular}

Note-In Experiment 1, the discrete fast was a 1 -sec rate and the discrete slow was a 4-sec rate. In Experiment 2, the discrete fast was a 2-sec rate and the discrete slow was a 6-sec rate. 
The results of the above comparisons differed depending on whether the two lists per condition were combined or the second list only was considered. For the combined lists, the complete condition significantly exceeded the discrete fast condition $[t(23)=2.92]$, but the difference between the two discrete conditions did not reach significance $[t(23)=1.67]$. The reverse results obtained for the second list comparisons, in which the two discrete conditions differed significantly $[t(23)=2.56]$, but the complete vs. discrete fast conditions did not $[\mathrm{t}(23)=1.44]$. The interpretation that we favor is that the faster presentation with list repetition is superior for free recall to the slower single list presentation, but that this superiority may depend upon some practice wherein the subject becomes accustomed to the rate and adopts an appropriate study strategy. What that strategy is cannot be specified at this point, but it is difficult to imagine that it involves much item elaboration or list organization. Interpretation of the complete vs. discrete fast comparison is more problematic, and we must conclude that although complete presentation is superior to discrete presentation at more or less standard rates, it may not be superior to discrete presentation at faster rates with list repetitions.

Experiment 3. The paired associate data are summarized in the bottom portion of Table 1. As with free recall, paired associate performance differed by presentation condition $[\mathrm{F}(2,44)=9.60, \mathrm{MSe}=4.19]$, and performance increased from the first to the second list within presentation conditions $[\mathrm{F}(1,22)=11.31$, MSe $=$ 6.77]. No other effects approached significance. The order of the means of the presentation conditions was identical to that in Experiment 2; the complete condition was highest and the 6-sec discrete condition, lowest. The difference between the two discrete conditions was significant $[t(23)=3.80]$, whereas the difference between the complete and $2-\mathrm{sec}$ discrete condition did not approach significance. It clearly was advantageous to present the list three times at the relatively rapid rate of $2 \mathrm{sec}$ compared with a single presentation at the slower 6-sec rate, despite the apparently greater opportunity for various mnemonic strategies (e.g., verbal or imaginal elaboration) with the slower presentation. Although complete presentation appears to offer even greater opportunities for such strategies, performance with the complete list did not differ from the rapid discrete condition.

\section{GENERAL DISCUSSION}

To summarize, in Experiment 1, complete presentation of study lists for free recall was superior to a single discrete presentation of the lists. The difference was large and pervasive. It was observed whether items occurred one, two, or three times within a list, whether multiply occurring items were massed or spaced, and whether discrete presentation was at a $2-, 4-$, or $6-\mathrm{sec}$ rate. Experiment 2 replicated the superiority of com- plete presentation to a single discrete presentation, and Experiment 3 extended this finding to paired associate learning. In both of these experiments, presentation of the complete list produced better recall than did the relatively slow successive presentation of the list a single time. However, Experiments 2 and 3 suggested that the superiority of complete presentation may not hold, or may be considerably less, when relatively fast presentation rates are used for discrete presentation and the list is presented more than once. Finally, Experiments 2 and 3 indicated that the latter procedure for discrete presentation probably is superior to a single, slower list presentation for both free recall and paired associate learning.

What accounts for the above findings and what are their theoretical implications? As we pointed out earlier, the superiority of complete presentation for free recall seems especially consonant with an organizational account of free recall learning. Such an account places heavy emphasis on the importance of detecting or establishing relationships among list items during retrieval. Complete presentation of the list should facilitate organization, since it makes it easy for the subject to inspect the total list for the presence of preexperimental relationships and to juxtapose mentally items from differing portions of the list for rehearsal. However, as pointed out in the introduction, studies that have manipulated the relatedness of words have not found that learning under the complete method has been enhanced, as would seem to be expected by an organizational approach.

An explanation that emphasizes elaboration of individual items does not appear to fit so well, since the emphasis is on the individual item rather than on its relationships to other list items. However, it may be argued that with complete presentation, the subjects are freer to allocate their elaborative encoding time more efficiently than with discrete presentation. For example, if a subject were to judge that a particular item will require greater elaboration than others, the complete presentation would permit the appropriate differential time allocation. Of course, the time allocation advantage might occur independently of one's concept of the particular nature of the encoding. That is, one might argue that with complete presentation, the subject is able to run quickly through the list, then back through again, giving spaced rehearsal to the items, and perhaps differential spaced rehearsal as well. Such an account differs from the first two in that there is no emphasis on strategic encoding operations concerning individual items. The only encoding strategy of any complexity would be in the decision to recycle through the list and (perhaps) to rehearse some items more than others.

Although an organizational theory accounts nicely for the superiority of complete presentation for free recall, it does not predict a similar superiority in paired associate learning. One could argue that the complete presentation is more effective for both for different 
reasons, so an organizational explanation for the complete-discrete difference in free recall would not be entirely ruled out by the paired-associate data. The remaining accounts seem to fare better. The paired associate data are compatible for the item elaboration account in much the same way as were the completediscrete free recall data. The assumption would be that some items require more time than most to establish strong associative links, perhaps by the use of mnemonics described by Rohwer (1973) as verbal or imaginal (imagery) elaboration.

However, neither the organization nor the elaboration notion are as easily reconciled with the differences between the discrete conditions. At least this seems true for those versions of organization and elaboration theories, and for any other theory, that place a heavy burden on the subject as a strategic encoder.

The amount of strategic processing that a subject can do with an item exposed for $1 \mathrm{sec}$ would seem to be minimal, even if there are several such exposures. Similarly, the generation of effective mnemonic links for paired associate learning seems unlikely to occur when both members of the pair are exposed together for a total of $2 \mathrm{sec}$. In each case, there seems to be opportunity for little more processing than is involved in the recognition of the items as particular words. Given the performance of the subjects in this condition relative to the complete and single slower discrete conditions, it seems reasonable to propose that effective encoding simply does not require the degree of strategic processing implied by a number of currently popular theoretical formulations. Note that our argument does not preclude the possibility that the subject behaves strategically with respect to list organization or item elaboration when the opportunity for such processing is provided, as it is with complete or slow discrete presentation. Subjects do so, although there is no evidence on this point from the present studies. What we do argue is that such processing evidently is not at the heart of effective encoding. Put another way, any formulation regarding processing necessary for good retention will have to take into account that such processing can occur with very little opportunity for the kinds of operations that some theories currently regard as critical.

Our own account of our results places major emphasis on the importance of repeated brief rehearsals of items. We regard the superiority of the fast to the slow discrete conditions as resulting primarily from the fact that the former provide spaced repetitions of the items, whereas slower single presentation is similar to massed presentation except that there is no off time between each presentation. To the extent that one accepts these conditions as functionally equivalent to those in the usual spacing experiment, the superiority of the discrete fast conditions is to be expected. It seems to us plausible that the relatively high levels of recall with complete presentation can be accounted for in a similar fashion.
That is, if the subject moves rapidly through the list, then recycles through the list, spaced presentation is functionally achieved. The subject may, in addition, vary the time spent from item to item, and that too may lead to more efficient processing of the list than does a constant rate. At this point, we can only speculate on the subject's behavior under the complete condition. All we can say with certainty is that whatever it is, it produces impressive amounts of learning in comparison with the presentation conditions ordinarily imposed by experimental psychologists.

The practical import of our results, and of further studies to refine and extend them, seems considerable. A good bit of instruction is machine controlled, and that seems likely to increase, given the increased availability of the low-cost microcomputers. Many such programs of instruction involve paced presentation of material to be studied, as with Atkinson's (1975) work with foreign language vocabulary, for example. A firm base of information regarding the optimal formats and rates/ frequencies for information presentation would seem mandatory for the efficient delivery of such instruction. For example, the present data suggest that the time allocated for the presentation of foreign language vocabulary pairs might be most efficiently used by presenting the list of pairs several times at more rapid rates than apparently are ordinarily used. For example, the rate of presentation in the Atkinson vocabulary instructional programs was on the order of $10 \mathrm{sec} /$ item in studies reporting the advantages of the key-word method for such instruction (Atkinson \& Raugh, 1975; Raugh \& Atkinson, 1975). Our data suggest that a much faster rate of presentation, and more presentations, might have resulted in considerably better learning. As it stands, the efficacy of mnemonic techniques that require slow presentation rates is likely to be overestimated in experiments wherein the control conditions also involve a very slow rate of presentation. Furthermore, complete presentation, when it is feasible, may be best of all.

\section{REFERENCES}

Anderson, J. R., \& REDER, L. M. An elaborative processing explanation of depth of processing. In L. S. Cermak \& F. I. M. Craik (Eds.), Levels of processing in human memory. Hillsdale, N.J: Erlbaum, 1979.

Atkinson, R. C. Mnemotechnics in second-language learning. American Psychologist, 1975, 30, 821-828.

Atkinson, R. C., \& RaUGH, M. R. An application of the mnemonic keyword method to the acquisition of a Russian vocabulary. Journal of Experimental Psychology: Human Learning and Memory, 1975, 104, 126-133.

Brewer, C. L. Presentation time, trials to criterion, and total time in verbal learning. Journal of Experimental Psychology, $1967,73,159-162$.

Brown, S. C., \& Brown, P. Comparison of successive and simultaneous methods of pair presentation in paired-association learning. Psychonomic Science, 1965, 2, 309-310.

Brown, S. C., \& READ, J. D. Effect of simultaneous list presenta- 
tion on paired-associate learning. Psychological Reports, 1966 , 19, 787-790.

Bugelski, B. R. Presentation time, total time, and mediation in paired-associate learning. Journal of Experimental Psychology, $1962,63,409-412$.

Cooper, E. H., \& Pantle, A. J. The total-time hypothesis in verbal learning. Psychological Bulletin, 1967, 68, 221-234.

Foote, R. A., \& Pollio, H. R. The role of associative organization in free recall under five different presentation conditions. Psychonomic Science, 1970, 20, 205-207.

Hall, J. W., Grossman, L. R., \& Elwood, K. D. Differences in encoding for free recall vs. recognition. Memory \& Cognition, 1976, 4, 507-513.

Imhoff, D. L., Horton, D. L., Weldon, L. J., \& Phillips, R. V. Rehearsal and processing capacity as factors in memory. Journal of Experimental Psychology: Human Learning and Memory, 1977, 3, 551-559.

Johnson, N. F. The functional relationship between amount learned and frequency vs. rate vs. total time of exposure of verbal materials. Journal of Verbal Learning and Verbal Behavior, 1964, 3, 502-504.

KNox, W. J., \& WofE, I. S. Learning of meaningful and nonmeaningful material by three methods of presentation. Psychological Record, 1965, 15, 513-520.

Lauer, P. A., Streby, W. J., \& Battig, W. F. The effects of alphabetic organization on the acquisition and delayed retention of semantically similar words. Journal of Experimental Psy. chology: Human Learning and Memory, 1976, 2, 182-189.

Light, L. L., \& Schurr, S. C. Context effects in recognition memory: Item order and unitization. Journal of Experimental Psychology, 1973, 100, 135-140.

Melton, A. W. The situation with respect to the spacing of repetitions and memory. Journal of Verbal Learning and Verbal Behavior, 1970, 9, 596-606.

Moursund, J. P., \& Chape, C. M. Comparison of three presentation methods in paired-associate learning. Psychonomic Science, 1966, 6, 167-168.

Mueller, J. H., Rankin, J. L., \& Carlomusto, M. Adult age differences in free recall as a function of basis of organization and method of presentation. Journal of Gerontology, 1979, 34, 375-380.

NoDine, C. F. Stimulus durations and total learning time in paired-associates learning. Journal of Experimental Psychology, $1965,69,534-536$.

Raugh, M. R., \& Atkinson, R. C. A mnemonic method for learning a second-language vocabulary. Journal of Educational Psychology, 1975, 67, 1-16.

RoHWER, W. D., JR. Elaboration and learning in childhood and adolescence. In H. W. Reese (Ed.), Advances in child development and behavior. New York: Academic Press, 1973.

Stubin, E. J., Heimer, W. I., \& TAtz, S. J. Total time and presentation time in paired-associate learning. Journal of Experimental Psychology, 1970, 84, 308-310.

Thonndike, E. L., \& LoRge, I. The teacher's word book of 30,000 words. New York: Columbia University Press, 1941.

(Accepted for publication February 17, 1981.) 